\title{
Editorial
}

\section{MEXICO 1978}

Now that the XI International Leprosy Congress has come and gone, many people will be asking awkward questions. Was it really worth it - the huge expenditure of money to bring together a thousand people? - the huge expenditure of effort and time for the detailed organization of the scientific sessions and the Workshops, the translation and printing of 333 abstracts in three languages, to say nothing of the social programme and the reservation of airplane seats and hotel rooms? Was it worth it? What was really accomplished? What will be its impact - if any — on the treatment and control of leprosy in the world? Will Mexico 1978 have any effect on the direction or content of leprosy research in the next five years?

The conventional stock-in-trade answers are unconvincing. Of course, "a good time was had by all". Of course, friend met friend, and had highly interesting conversation. Of course, like-minded people got together to discuss common problems. But with the long-distance telephone and air mail, is any additional benefit likely to accrue to the sufferer from leprosy or to those at risk of catching leprosy, from the masses of paper and the deafening Babel of multilingual communications?

Let us frankly admit that no unexpected or dramatic "breakthrough" was announced at Mexico. In any case, sober scientists do not usually conceal their research findings until they can allow them to burst upon a waiting world on such an occasion as an International Congress. Mostly, progress is made by the accumulation of small bits and pieces in which some genius will eventually discern a recognizable pattern.

Thus, in experimental leprosy, the immunologically deficient mouse has been joined by the armadillo, the nude mouse, the thymectomized rat, the hedgehog and the Korean chipmunk. Unimaginable quantities of $M$. leprae are now required, and obtained, by the World Health Organization cooperative IMMLEP programme, and progress is reported on the analysis of the complicated antigenic structure of cell-walls and cytoplasm. Interesting, and potentially important immunological comparisons between $M$. leprae and its nearest relations are being made, and further steps taken along the path that must lead to the making, appraisal and evaluation of specific skin tests and eventually to a specific protective vaccine. Perhaps some addition to, or modification of classical BCG vaccine will serve the purpose.

In microbiology, work was reported on the fascinating possibility that strains of organisms obtained from human lepromatous material may develop acid- and alcohol-fastness in culture. Over the years, successive claims to successful growth in artificial culture media have failed to find confirmation, and the use of the many accepted criteria for identification now available make the task of exclusion more precise and less difficult. In this connection, it is 
now recognized that the addition of hyaluronic acid to special media may facilitate and enhance the growth of several mycobacteria, but "not proven" must be the verdict of some recent claims. The use of semi-solid agar, pyruvate, mycobactins and other possible adjuvants indicates the continuing interest in attempts to culture $M$. leprae.

Other important matters in this field were aired in Mexico, if not finally decided to the equal satisfaction of all interested parties. The occurrence of a leprosy-like disease, caused by a $M$. leprae-like organism, in wild armadillos in the southern United States is now generally admitted, and further work should indicate the epidemiological importance of this potential reservoir of infection, as well as confirm the intrinsic immunological interest of the armadillo.

The excellent investigations reported both at the scientific sessions and the pre-Congress Workshops, and the general air of scientific keenness and enthusiastic pursuit of new knowledge were met by no corresponding optimism by the clinicians and therapists. Here, the atmosphere was not quite one of unrelieved gloom and despondency, but certainly there was an air of soberalmost sombre - realism as the twin spectres of drug-resistance and persister organisms were cited and examined. Despite the availability for 30 years of an effective-and cheap-anti-leprotic drug, mycobacteriostatic (if not mycobactericidal) in standard therapeutic doses, an insufficient impact has been made on the worldwide prevalence of leprosy. The conclusions and recommendations of the WHO 5th Expert Committee on Leprosy were endorsed, and the recommendations of the LEPRA "Heathrow Report" were put forward as a series of practical measures for recognizing, treating and preventing drug resistance. Participants from countries where leprosy is most prevalent, countries facing other and more urgent problems of transmissible disease and nutrition, were in general far from hopef ul that leprosy would be taken seriously enough by their governments (or by interested voluntary agencies) and resources made available to counter these two threats. Both the Workshop and the scientific session emphasized the urgent necessity for controlled trials of combinations of drugs, and for chemical modifications of some existing drugs to enhance their anti-leprotic activity. Knowledge is urgently needed on the most effective-and least costly — way to administer rifampicin, for instance; otherwise this valuable drug may be so misused that its potential wide usefulness may be seriously impaired.

Newer treatments designed to modify the immunological response, temporarily or for longer periods, by introducing substances like transfer factor, or clones of lymphocytes derived from healthy donors, or donors suffering from tuberculoid leprosy, or such products as levamisole, have given discordant or equivocal results, and no general recommendations are as yet forthcoming.

It is in this area of common interest that the clinicians and those responsible for programmes for leprosy control met the immunologists who are elucidating the complex antigenic structure of $M$. leprae and the unexpectedly complicated pattern of cellular and humoral immunity. The subject of immunology attracted more papers than any other, an indication of the widespread interest in and importance of leprosy as an investigative and demonstrative model. The 
techniques of crossed immuno-electrophoresis and radio-immune-assay are being used in several laboratories as tools for identifying the antigenic patterns of $M$. leprae and related mycobacteria. Although at first sight this highly specialized and sophisticated type of investigation may appear to be far removed from the problems confronting the field worker in his day-to-day contacts with the defaulting patient or the uncooperative contacts, or the breakdown in the supply of dapsone-yet the solution of these questions, or perhaps the pursuit of "leads" arising during current investigations, will eventually provide the man-in-the-field with more effective weapons and strategies with which to attempt to treat and control leprosy.

Several reports were presented of the relation between skin tests with various antigens and past infection with leprosy, with active or quiescent disease, and with disease characterized by different degrees-or complete absence-of cell-mediated immunity. The search for the specific immune defect in patients with lepromatous leprosy continues, as well as the search for some field test that would readily and reliably (and inexpensively) identify those individuals who have this defect, and especially those individuals who are possibly exposed to viable $M$. leprae in the household or community. New studies are being made of the results of skin testing with armadillo-derived lepromin and human lepromin. A practical aspect of these studies is the light they may shed on the variable results of adequately controlled BCG prophylaxis programmes; it may be that previous exposure to opportunist mycobacteria may account for the difference in skin sensitization and protection rates afforded in the well-known trials.

The possible transmissible or hereditary factor in susceptibility to leprosy continues to baffle investigators. Blood groups, genetic markers, and now HLA (histocompatibility complex) antigen patterns do not provide any more than indications of present ignorance and future possible avenues of research. The well-known clustering of leprosy in families, and the reported apparent refractoriness of $95 \%$ of spouses of adult leprosy patients, provided the baselines for much theorizing and little solid progress.

The epidemiometric model recently developed is proving its usefulness and adaptability. In particular, the inputs concerning dapsone resistance, subclinical infections and persister viable organisms are modifying the whole picture of leprosy control. In the light of the increasing population in countries where leprosy is still virtually uncontrolled, the emerging picture is likely to cause deepening concern about the effectiveness of our present methods of control.

An upsurge of interest in nerve damage in leprosy is apparent in many quarters. The pathophysiologists are now in broad agreement concerning the main features of nerve function and malfunction in leprosy, and the pathogenesis and triggering mechanisms are now better understood. The relative importance of the various possible mechanisms, operative in the individual patient, merit more investigation. The relative importance of temperature, entrapment, trauma, immunological factors (such as immune complexes and complement) and possible sensitizing phenomena (like biochemical products derived from damaged nerve fibres or degenerating 
mycobacteria)—will receive increasing attention from investigators approaching the subject from diverse standpoints. It was suggested that the whole subject of nerve damage in leprosy demands a full scientific session at the next Congress.

The role of surgery in the relief of nerve pain and the prevention of further nerve damage was studied in several well-documented papers, but such delicate surgery, it was urged, should be practised by medically-minded surgeons, and not by dabbling physicians. Convincing results of nervedecompression, when practised for precise indications in appropriate surroundings, were presented. In this matter of reconstructive and plastic surgery, it was emphasized that the patient whose deformity or incapacity was due to leprosy should not suffer any discrimination on this account, but should wherever possible be admitted to a general ward. Yet reconstructive surgery in a leprosy patient demands high standards of knowledge, judgement and operative skill.

The rehabilitation of the sufferer from leprosy was sympathetically considered in more than one session. The surgeons and the social workers were as concerned as the clinicians in charge of leprosy programmes. Some were of the opinion that the abolition of stigma and the equal treatment of leprosy sufferers could be achieved only by the complete integration of leprosy into the health programmes of government, while others were equally convinced that integration would mean that the leprosy patient would cease to receive even the treatment and consideration he now enjoys in many "vertical" programmes. All were agreed, however, that education in its broadest sense was the common ground that must provide the essential sine qua non of any attempt to treat leprosy sufferers humanely and to ensure that all who needed help - medical, surgical, financial, social - could be sure of having access to it. The vicious circle of fear, stigma and prejudice can be interrupted only by effective health education.

The word "leprosy" and its derivatives came in for some critical examination. The crux of the matter is that in most linguistic groups, the real sting of leprosy resides in the disease and not in the name by which it is known. The members of the sessions on social aspects of leprosy faced this problem seriously in the course of the well-attended meetings, and the hope was expressed that some of the lingering old-style leprosaria might be utilized for the care of those disabled from whatever cause.

Not only in the special pre-Congress Workshop, but underlying many of the sessions on many diverse aspects of leprosy, was the urgent and continuing necessity for training for everybody concerned with leprosy; doctors and nurses, physiotherapists and laboratory technicians, and especially the auxiliary worker who is the keystone in most effective leprosy control programmes. Standardization of teaching materials is not nearly so important as the provision and availability of materials appropriate and adapted to the local needs of the situation, in language, pictorial or diagrammatic presentation, in content and in the down-to-earth practical nature of booklets, audiovisual aids, demonstrations, etc. The voluntary agencies were urged to continue their much-appreciated contribution, in this area, to the present 
worldwide leprosy campaign, and to enlist the cooperation of technically qualified specialists to ensure the didactic acceptability of the materials they were publishing and disseminating.

A word must be said about the poster presentations. Despite initial misgivings on the part of some, it was evident that many authors welcomed the opportunity to present their work in this form, just as they welcomed the chance of discussion with interested individuals, unstressed by the demands of platform presentation within a strictly limited time.

Perhaps there were too many papers at Mexico, too many people, too little time for discussion. Perhaps there was a tacit acknowledgement that sophisticated research gains a greater popular rating than the humdrum activities of a good leprosy control programme. But research is the lifeblood of leprosy, and unless we can make available to the many the privileges of diagnosis, care and treatment enjoyed by the few, our leprosy programmes in the future will achieve no better results than they have in the past.

Where do we go from here?

I detect a note of sober-even sombre-realism in the papers and sessions. We are learning more of the complexity of the leprosy organism and of the immune response to challenge. And the twin spectres of drug resistance and persistently viable organisms dominate much of our thinking today. But I can also discern an excitement, an enthusiasm, as unforeseen and unimagined vistas of research are opening up to the research immunologist and microbiologist.

Coupled with the realism and the excitement, can we not all see, and welcome, the increasing interest in the whole social environment of the sufferer from leprosy? $\mathrm{He}$ is a fellow human-being, a man (or woman) like unto ourselves, with hopes and frustrations, with family contacts, with needs for food and housing and employment and the simple joys of life.

In this Congress, we met each other, and appreciated each other's work. And we are coming to realize, whatever our particular field of activity, that we need each other more than ever before as we face the common foe.

Highlights? Yes, a few. More importantly, a general intense glow of interest and cooperate effort, a warmth of mutual appreciation and understanding, and a realization of our interdependency in the One World, the global community. Coupled with all this, is the working together of the research scientist, the concerned therapist, and those deeply moved by the human plight of the sufferer from leprosy.

This spirit augurs well for the future-whatever the serious problems we may have to face. Let us put into practice what we already know, and strive after new knowledge that will help solve this intractable and challenging problem. 